\title{
Long non-coding RNA HOXB-AS1 promotes proliferation, migration and invasion of glioblastoma cells via HOXB-AS1/miR-885-3p/HOXB2 axis
}

\author{
X. CHEN ${ }^{1}$, L. Q. LI ${ }^{2}$, X. QIU ${ }^{3}, H . W U^{1, *}$ \\ ${ }^{1}$ Department of Neurosurgery, The Affiliated Hospital of Zunyi Medical College, Zunyi, China; ${ }^{2}$ Central Clinical Laboratory, Shenzhen Hospital, \\ Southern Medical University, Shenzhen, China; ${ }^{3}$ The Clinical Medical College of Tianjin Medical University, Tianjin, China
}

${ }^{*}$ Correspondence: wht0327@163.com

Received June 6, 2018 / Accepted November 21, 2018

\begin{abstract}
Long non-coding RNAs (lncRNAs) have been proven to play important roles in carcinogenesis and development of numerous cancers, but their biological functions in glioblastoma remain largely unknown. In this study, we found that HOXB-AS1 was highly expressed in human glioblastoma tissues and cell lines, and was associated with survival time of patients. Further analysis showed that knock-down of HOXB-AS1 inhibited cell proliferation via inducing S phase cell cycle arrest and suppressed migration and invasion ability of cells. Mechanism study revealed that HOXB-AS1 is mainly located in cytoplasm and functions as competing endogenous RNA via sponging of miR-885-3p. Moreover, inhibition of miR-885-3p antagonized the effects of HOXB-AS1 knock-down and promoted proliferation, migration and invasion of glioblastoma cells. Finally, we found that sponging of miR-885-3p by HOXB-AS1 could further affect the expression of HOXB2. Taken together, we demonstrate that HOXB-AS1/miR-885-3p/HOXB2 axis regulates proliferation, migration and invasion of glioblastoma cells and can serve as a potential biomarker for the malignancy.
\end{abstract}

Key words: glioblastoma, HOXB-AS1, miR-885-3p, proliferation, migration

Glioblastoma (GBM) is the most common and aggressive form of primary brain tumors with an incidence of $3-4 / 100,000$ per year $[1,2]$, with a median overall survival (OS) of approximately 15 months, and only $3-5 \%$ of patients survived longer than 3 years $[3,4]$. In the treatments of GBM patients, maximal surgical resection, radiotherapy, and chemotherapy with temozolomide (TMZ) remain the most standard options, which have been demonstrated to significantly improve the survival time [5]. However, the high speed growth of tumor, invasive behaviors, and resistance to radioand/or chemotherapy are the main causes leading to the poor prognosis of GBM patients [6, 7]. Accordingly, it is of great significance to have a better understanding of the carcinogenesis and progression of GBM, which could provide new biomarkers and therapeutic targets for clinical diagnosis and treatment of GBM patients.

Long non-coding RNAs (lncRNAs), which are more than 200 nucleotides in length, are a recently discovered novel class of genes with regulatory functions but lacking proteincoding ability. Increasing evidence has revealed that dysregulated expression of lncRNAs is involved in tumor initiation, progression, and metastasis $[8,9]$. Numerous studies have proven that lncRNAs could function via different mecha- nisms including $\mathrm{X}$ chromosome inactivation, splicing, imprinting, epigenetic control, gene transcription regulation, and sponging microRNAs [10-14]. As one of the most important mechanisms, sponging microRNAs and function as a competing endogenous RNAs (ceRNAs) has shown critical roles in different cancers $[15,16]$. Yang et al. reported that MCM3AP-AS1 could directly sponge miR-211 and promote glioblastoma angiogenesis [15]. LncHERG was reported to promote cell proliferation, migration, and invasion in glioblastoma [16]. All the studies indicated that lncRNAs could affect the carcinogenesis and malignant behaviors of glioblastoma, which further influence the prognosis of GBM patients. While the functions and mechanisms of lncRNAs remain largely unknown, it of great significance to explore novel lncRNAs and to identify their functions, which might provide potential therapeutic targets for clinical treatments of GBM patients.

In this study, a newly identified lncRNAs termed HOXB-AS1, which has not been investigated previously, was reported. We investigated the tumor promoting roles of HOXB-AS1 via direct sponging of miR-885-3p and further affecting oncogene HOXB2. The research broadens our insights into the underlying mechanisms in the proliferation, 
migration and invasion of GBM cells, and provides a new therapeutic target for GBM patients in clinic.

\section{Materials and methods}

Low grade glioma and GBM RNA sequencing data. The RNA-seq data of 486 low grade glioma (LGG) and 154 glioblastoma (GBM) tissues were downloaded and analyzed based on the Atlas of Noncoding RNAs in Cancer (TANRIC) database [17].

Cell lines and cell culture. HA, LN229, U87 and U251 cell lines (ATCC, Rockville, MD, USA) were cultured in Dulbecco's modified Eagle's medium supplemented with $10 \%$ fetal bovine serum (Aurogene) in a humidified atmosphere containing $5 \% \mathrm{CO}_{2}$ at $37^{\circ} \mathrm{C}$.

Construction of stable HOXB-AS1 knock-down cell lines. HOXB-AS1 specific shRNA vector and its control were acquired from Vigene Biosciences (Rockville, USA) and were transfected into U87 and U251 cell lines using Lipofectamine $^{\circledast}$ RNAiMAX Reagent (Thermo Fisher Scientific, Inc.) according to the manufacturer's protocol. The shRNA sequence to HOXB-AS1 was 5'-GGAAGAGGATTGTGAAGTTTA-3'.

Cell viability assay. Cell viability was determined by a 3-(4, 5-dimethylthiazol-2-yl)-2, 5-diphenyltetrazolium bromide (MTT) assay for 6 days. $20 \mu \mathrm{l}$ of MTT $(5 \mathrm{mg} / \mathrm{ml}$ in PBS) was added into each well and incubated for $4 \mathrm{~h}$. The supernatants were carefully aspirated, and $100 \mu \mathrm{l}$ of dimethyl sulfoxide (DMSO) was added to each well. Absorbance values at $490 \mathrm{~nm}$ were measured on a Microplate Reader (Bio-Rad). Ethynyl deoxyuridine (Edu) assays was performed as previously described [18].

Cell apoptosis assay. Cell apoptosis was detected using an Annexin V-FITC/PI apoptosis detection kit (JingMei Biotech, Beijing, China) as previously described [19]. Briefly, cells were treated with EDTA-free trypsin, and resuspended with $400 \mu \mathrm{L}$ Annexin binding buffer at a concentration of $10^{6}$ cells $/ \mathrm{ml}$. Then, $5 \mathrm{ml}$ FITC-conjugated AnnexinV and $10 \mathrm{ml}$ PI were added to the cells and incubated at room temperature for $15 \mathrm{~min}$ in dark. Cell apoptosis assay was performed within $1 \mathrm{~h}$ post-staining on a flow cytometry.

Cell cycle assay. Cells were collected, washed twice with $1 \mathrm{x}$ PBS, and fixed in $70 \%$ ethanol at $-20^{\circ} \mathrm{C}$. After $24 \mathrm{~h}$ of fixation, cells were incubated with RNase A (Takara Bio, Inc., Otsu, Japan) at $100 \mu \mathrm{g} / \mathrm{ml}$ in $1 \mathrm{X} \mathrm{PBS}$ for $30 \mathrm{~min}$ at $37^{\circ} \mathrm{C}$. Cells were then stained with propidium iodide (PI; BD Biosciences, San Jose, CA, USA) at $50 \mu \mathrm{g} / \mathrm{ml}$ for $30 \mathrm{~min}$ at room temperature. Subsequently, cells were analyzed for DNA content using a BD FACSCalibur flow cytometer (BD Biosciences).

Wound healing assay. Cells were harvested and reseeded into 96-well culture plates at the concentration of $3 \times 10^{4}$ / well and $2 \times 10^{4} /$ well, respectively. After $16 \mathrm{~h}$ incubation, when cells had reached more than $90 \%$ convergence, the wound was performed, washed twice in PBS, replaced with serum-free medium, and maintained in an incubator. Cells were photographed after 0,8 , and $24 \mathrm{~h}$, and the width of the wound was recorded as the wound distance.

Transwell assay. The cell migration ability was analyzed by a transwell assay, which was performed in 24-well transwell chambers (Corning, NY, USA). After $24 \mathrm{~h}$ transfection, cells in $200 \mathrm{ml}$ serum-free medium were reseeded into the upper chamber. The lower chamber was filled with $600 \mu \mathrm{L}$ medium with $20 \%$ FBS. After incubating, cells on the inner membrane were removed. The outer membrane was fixed with $4 \%$ paraformaldehyde and stained with $0.1 \%$ crystal violet solution. The invasion assay was performed in the same way as the migration assay except that the membrane was coated with matrigel (BD Biosciences, Bedford, MA, USA).The cells were observed under a microscope and five fields were randomly selected to be photographed.

Dual-luciferase reporter assay. Dual-luciferase miRNA Target Expression vector pmirGLO (Promega, Madison, WI, USA) was used to generate luciferase reporter constructs. Full length HOXB-AS1 sequences with wild type (Wt) or mutant type (Mut) microRNA binding sites were obtained from Vigene Biosciences (Rockville, USA). Cells were seeded in 96-well plates and co-transfected with wild-type or mutated HOXB-AS1 constructs and miR-885-3p mimic. Luciferase activity was measured with the dual-luciferase reporter assay system (Promega). Firefly luciferase activity was normalized against Renilla luciferase activity.

RNA immunoprecipitation (RIP) assay. RIP experiments were performed using the Magna RIP RNA-Binding Protein Immunoprecipitation Kit (Millipore, Billerica, MA, USA) according to the manufacturer's instructions. Antibodies for RIP assays of AGO2, or control IgG were purchased from Millipore. The coprecipitated RNAs were detected by qRT-PCR. The total RNAs were the input controls.

Quantitative RT-PCR analysis. Total RNA was extracted from cells using the TRIzol reagent (Invitrogen, Carlsbad, CA, USA). PrimeScript Reverse Transcriptase (RT) Reagent Kit (TaKaRa, Shiga, Japan) was used to synthesize cDNA from total RNA. MiRNA from $1 \mu \mathrm{g}$ of total RNA was reverse transcribed using the Prime-Script miRNA cDNA Synthesis Kit (TaKaRa). Real-time PCR was performed on Applied Biosystems StepOne plus System, and results were analyzed as previously described [20]. The primers used in our study included: HOXB-AS1 F: GGGGACTCCAGCGAAAT; HOXB-AS1 R: ACCCGAAGCCCAACCAC; GAPDH F: GTCTCCTCTGACTTCAACAGCG; GAPDHR: ACCACCCTGTTGCTGTAGCCAA; MiR-885-3p: AGGCAGCGGGGTGTAGTGGATA; U6: GCGCGTCGTGAAGCGTTC.

Western blot analysis. Western blot analysis was performed as described previously [17]. In brief, cells were harvested and lysed in lysis buffer containing protease inhibitors. Subsequently, $50 \mu \mathrm{g}$ of total cellular protein from each sample were separated by $10 \%$ SDS-PAGE and electrotransferred onto polyvinylidene fluoride (PVDF) membrane 
using a semi-dry blotting apparatus (Bio-Rad, Hercules, CA, USA). The membranes were blocked with $5 \%$ nonfat milk at room temperature for $1 \mathrm{~h}$, and then incubated with primary antibodies overnight at $4^{\circ} \mathrm{C}$. After incubation with the appropriate secondary antibodies, the protein bands were detected using the Pro-lighting HRP agent. Expression of $\beta$-actin was used as a loading control. The antibody used in this study was obtained from Abcam: HOXB2 (ab220390), b-Actin (ab8226).

Statistical analysis. All data are presented as means \pm S.D. from at least three independent experiments. The software SPSS V18.0 was used for statistical analysis. Statistical significance of differences between two groups was evaluated using
Student's t-test, and one-way ANOVA was used to determine the significance of differences among multiple groups. Differences with $\mathrm{p}<0.05$ were considered statistically significant.

\section{Results}

LncRNA HOXB-AS1 was up-regulated in GBM and associated with prognosis. To explore potential functional lncRNAs associated with GBM, the lncRNA expression data and clinical information of LGG and GBM patients were downloaded and analyzed, and a total of 486 LGG and 154 GBM patients were involved (Figure 1A). Compared with LGG tissues, 199 lncRNAs were upregulated and $185 \operatorname{lncRNAs}$

A
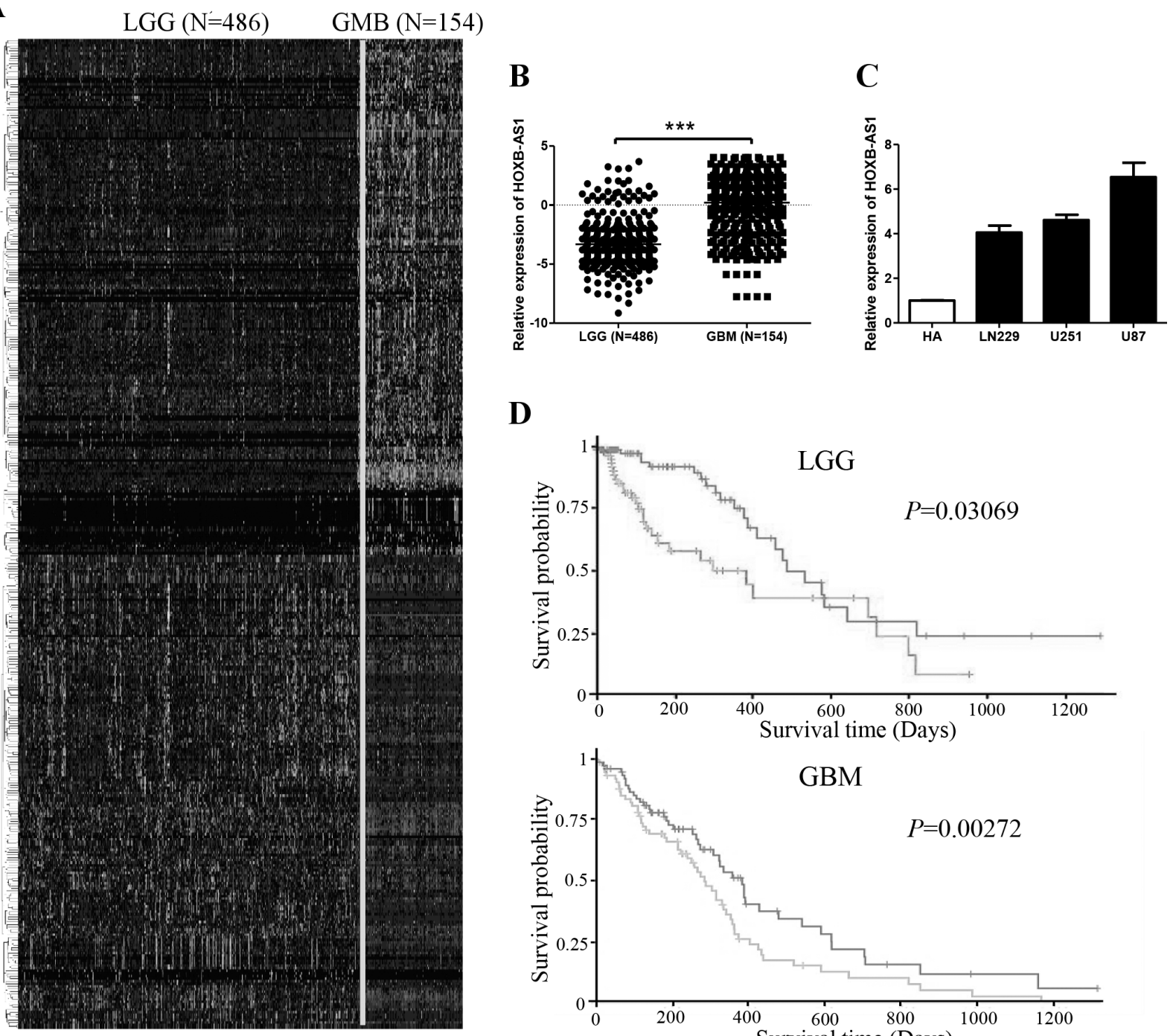

D
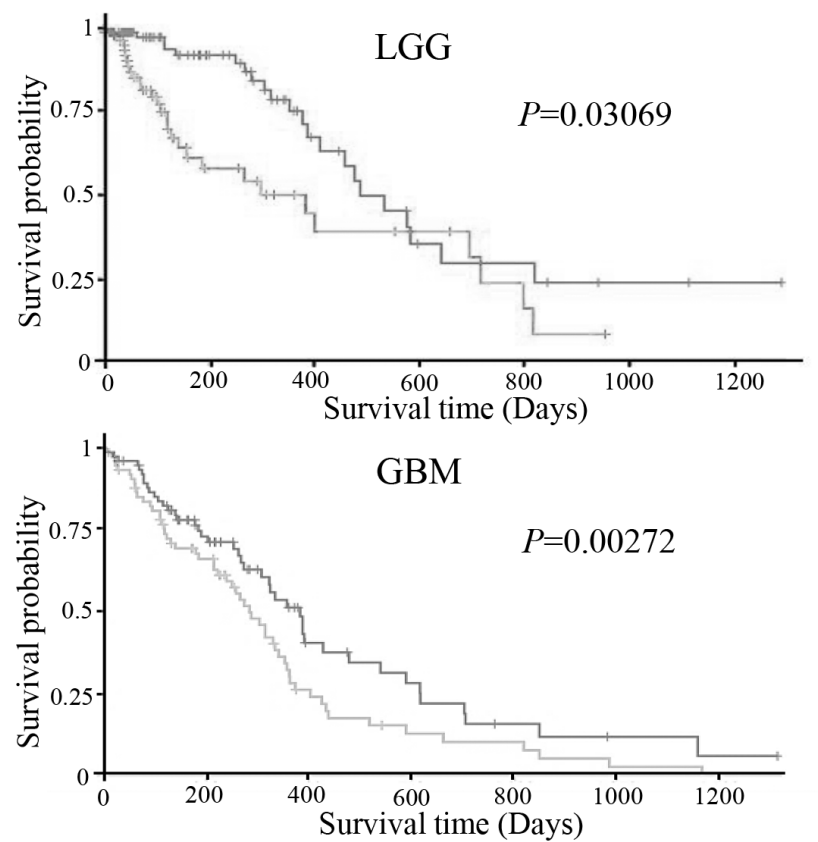

Figure 1. LncRNA HOXB-AS1 was up-regulated in GBM and associated with prognosis. A) Heat map diagram depicted the expression of significantly dysregulated lncRNAs in GBM compared with LGG. B) Expression of HOXB-AS1 in GBM was significantly higher compared with LGG. C) Expression of HOXB-AS1 in normal HA cell lines and GBM cell lines. D) High expression of HOXB-AS1 predicted poorer prognosis in both LGG and GBM based on Kaplan-Meier analysis. ${ }^{* * *} \mathrm{p}<0.001$. 
were downregulated in GBM patients, and HOXB-AS1 was one of the most significantly up-regulated lncRNAs. The expression of HOXB-AS1 in both LGG and GBM tissues are shown (Figure 1B). We then examined the expression of HOXB-AS1 in human astrocyte (HA) cell line and human glioma cell lines (LN229, U87 and U251), and a remarkable up-regulation of HOXB-AS1 was found in human glioma cell lines (Figure 1C). Also, we found that the overexpression of HOXB-AS1 contributed to poorer prognosis in both LGG and GBM patients based on TCGA database (Figure 1D). In conclusion, our results indicated that HOXB-AS1 might be a potential regulator and play important roles in the regulation of the carcinogenesis and progression of GBM.

Knock-down of HOXB-AS1 suppressed proliferation of GBM cells via inducing $S$ phase cell cycle arrest. It is well-known that rapid proliferation contributes to fast progression of neoplasms. As we had explored that HOXB-AS1 was highly expressed in GBM tissues as well as cell lines, we further wondered whether HOXB-AS1 was involved in the proliferation of GBM cells. To validate the proliferation effects of HOXB-AS1, we firstly knocked down HOXB-AS1 in both U87 and U251 and stabilized cell lines were established (Figure 2A). As shown in Figure 2B, knockdown of HOXB-AS1 could significantly inhibit the proliferation of both cell lines. Moreover, Edu assay revealed that down-regulation of HOXB-AS1 decreased the proliferation activity (Figure 2C). To further explore the mechanism, we performed cell cycle assay using flow cytometry, and the cell cycle was proved to be arrested in $\mathrm{S}$ phase in both cell lines (Figure 2D).

Previous studies reported that the cell inhibition can be induced not only by cell cycle arresting, but also by cell death including cell apoptosis [21, 22]. Here, we performed cell apoptosis assay to evaluate the effects of LncRNA HOXB-AS1 on cell apoptosis. As shown in Supplementary Figure 1, we found no significant changes in cell apoptosis of both U87 and U251 cells after knock-down of HOXB-AS1, indicating that the proliferation suppression effect was mainly caused by cell cycle arrest. In conclusion, our findings demonstrated that knock-down of HOXB-AS1 suppressed proliferation of GBM cells via inducing $S$ phase arrest.

Knock-down of HOXB-AS1 suppressed migration and invasion abilities of GBM cells. Previous studies suggested that the metastasis of GBM was one of the most important factors that influence the prognosis of patients [23]. Some genes such as NEK2and microRNA-506, play important roles in both proliferation, migration and invasion of tumors, indicating a potential correlation between proliferation, migration and invasion $[24,25]$. To further evaluate the influence of HOXB-AS1 knock-down on the migration and invasion of GBM cells, we performed transwell assay and wound healing assay. As shown in Figure 3A, the transwell assay indicated that down-regulation of HOXB-AS1 could significantly suppress the migration of U87 and U251 cells. And we also proved that silencing of HOXB-AS1 inhibited the invasion abilities of cells (Figure 3B). Furthermore, we found that the wound was much wider in the knock-down group after scratching compared with control group in both cell lines (Figure 3C). Based on above evidences, our results suggested that HOXB-AS1 could regulate the migration and invasion abilities of GBM cells.

HOXB-AS1 functioned as ceRNA via directly sponging miR-885-3p. After showing that knock-down of HOXB-AS1 could significantly suppress the proliferation, migration and invasion of GBM cells, we further explored the mechanisms. Previous studies reported that the possible mechanism of lncRNAs was closely associated with the subcellular locations of lncRNAs in cells [13]. We firstly examined the subcellular localization of HOXB-AS1 in GBM cells. As shown in Figure $4 \mathrm{~A}, \mathrm{HOXB}-\mathrm{AS} 1$ was mainly located in the cytoplasm of both cell lines, which indicated that HOXB-AS1 could function as ceRNA via directly sponging microRNAs. Further bioinformatic analysis found a potential binding site of miR-885-3p on HOXB-AS1 sequence (Figure 4B). To validate the hypothesis, we constructed full length HOXB-AS1 sequence with wild type (Wt) and mutant type (Mut) microRNA binding site into pMIR-GLO vector (Figure 4C). As shown in Figure $4 \mathrm{D}$, the luciferase reporter assay revealed the directly target of miR-885-3p on HOXB-AS1, as the relative luciferase activity was remarkably decreased by miR-885-3p in $\mathrm{Wt}$ group and showed no obviously change in Mut group. It has been reported that Ago2 is a key component of RNA-induced silencing complexes (RISC) [26], and we used an anti-Ago2 antibody to perform RNA immunoprecipitation (RIP). The result showed that both HOXB-AS1 and miR-885-3p were enriched with anti-Ago2 antibody compared with IgG (Figure 4E). Finally, we showed that the expression of miR-885-3p was significantly overexpressed after knockdown of HOXB-AS1, which further demonstrated the combination of HOXB-AS1 with miR-885-3p (Figure 4F). Overall, our results demonstrated that HOXB-AS1 could function as ceRNA via direct sponging miR-885-3p.

HOXB-AS1 promoted the proliferation, migration and invasion of GBM cells via HOXB-AS1/miR-885-3p/ HOXB2 axis. The above results proved that HOXB-AS1 could promote the proliferation, migration and invasion of GBM cells, and can act as a ceRNA via directly sponging miR-885-3p. We then explored whether the functions of HOXB-AS1 could be rescued by miR-885-3p. As shown in Figure 5A, the expression of miR-885-3p was verified after HOXB-AS1 knock-down and miR-885-3p inhibition. MTT assay showed that the inhibition of miR-885-3p by inhibitor could reverse the proliferation suppression effect induced by HOXB-AS1 knock-down in U87 and U251 cell lines (Figure 5B). Furthermore, transwell assay suggested that inhibition of miR-885-3p could rescue both migration and invasion inhibition effects (Figure $5 \mathrm{C}$ ). These results demonstrated that HOXB-AS1 promoted the proliferation, migration and invasion of GBM cells via inhibition of miR-885-3p. 
A

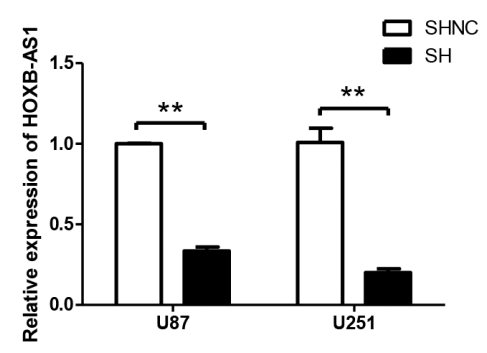

$\mathrm{C}$
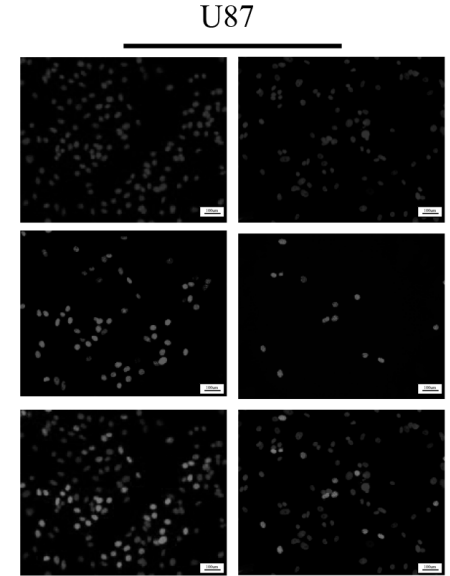

sh-NC

$D$

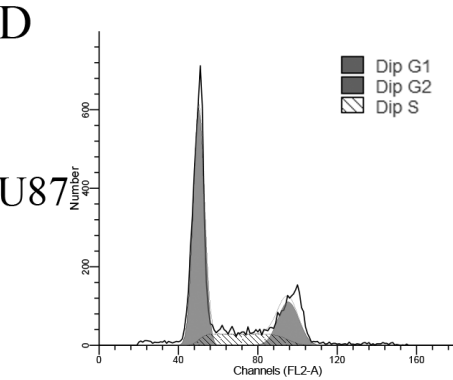

sh-NC

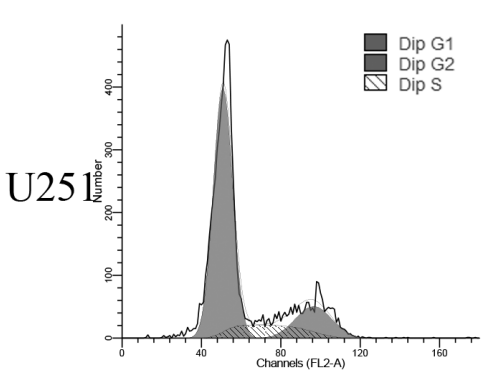

sh-NC
B
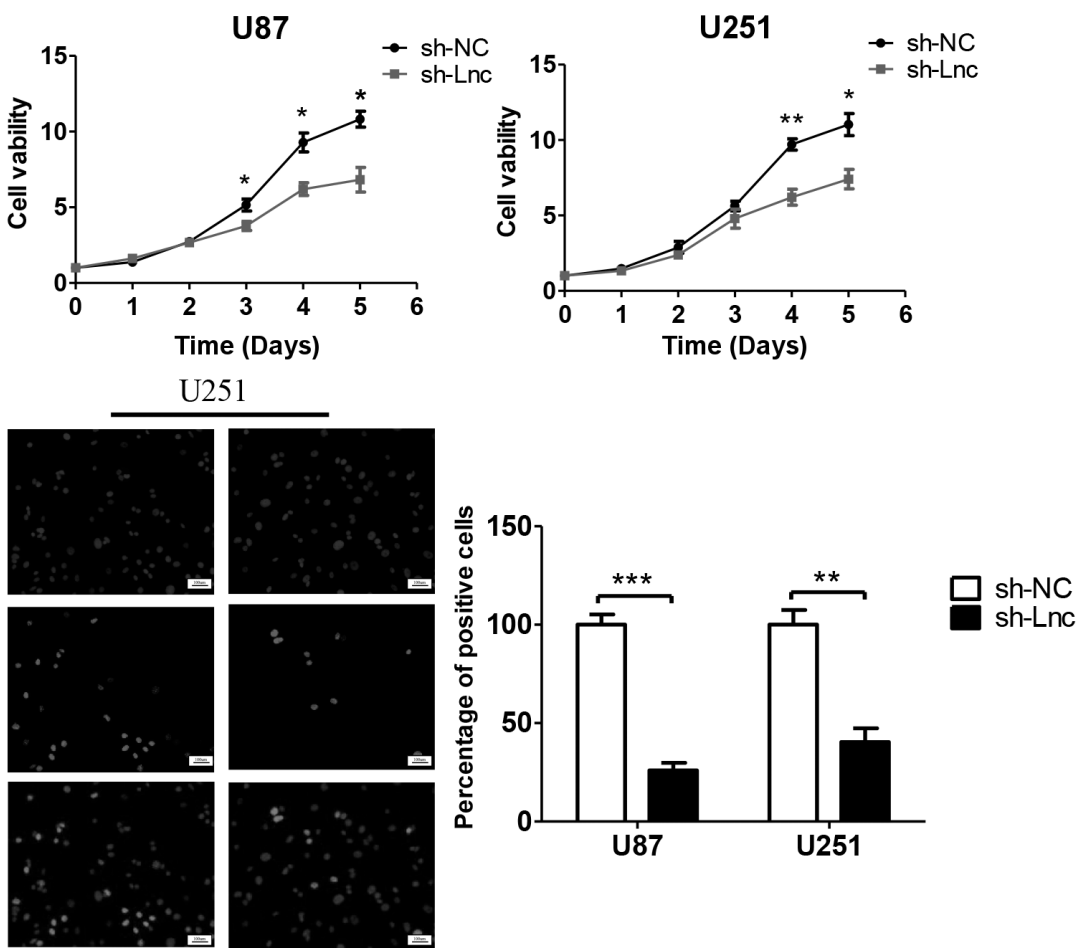

sh-Lnc

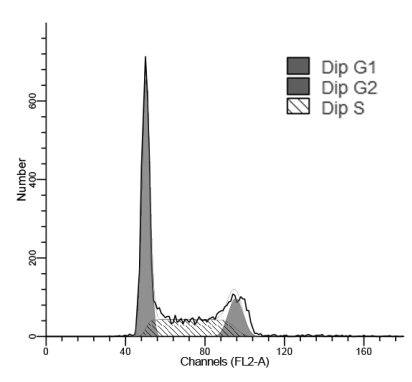

sh-Lnc
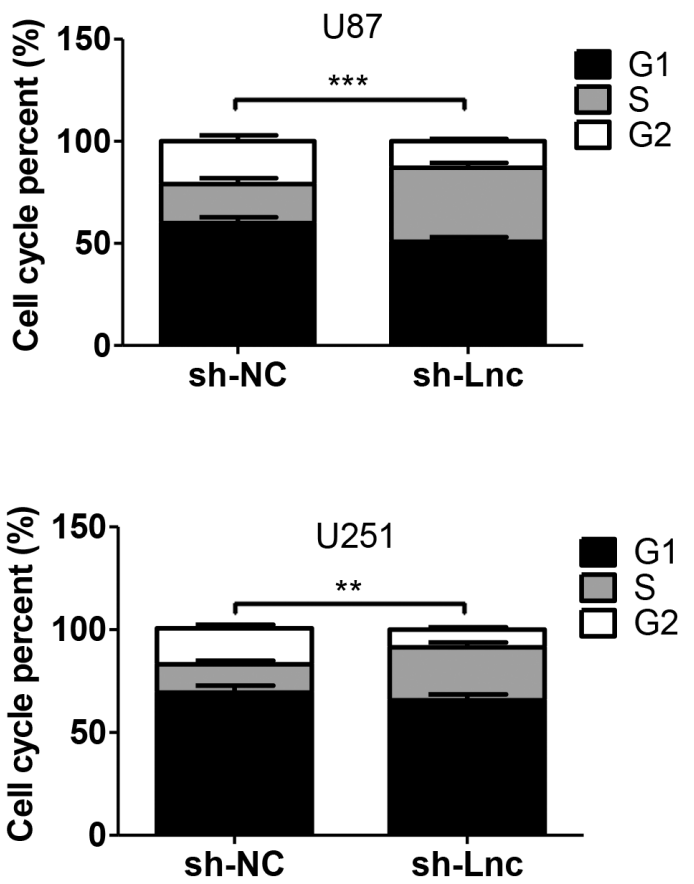

sh-Lnc

Figure 2. Knock-down of HOXB-AS1 suppressed proliferation of GBM cells via inducing S phase arrest. A) Both U87 and U251 cell lines were transfected with sh-NC or sh-Lnc, and the efficiency of HOXB-AS1 knock-down was evaluated. B) Cell viability was measured by MTT assay in U87 and U251 cell lines, and cell viability of cells with HOXB-AS1 knock-down was significantly inhibited. C) Edu assay was performed to examine the proliferation activity of cells, and data showed remarkably reduced proliferation activity with HOXB-AS1 knock-down. D) The cell cycle of U87 and U251 cells was examined by flow cytomerty, and data indicated $S$ phage cell cycle arrest after HOXB-AS1 knock-down. ${ }^{*} p<0.05,{ }^{* *} p<0.01,{ }^{* * *} p<0.001$. 
A

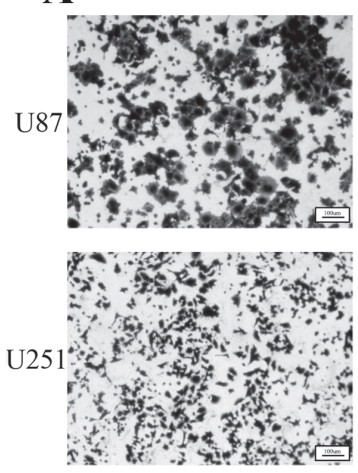

sh-NC

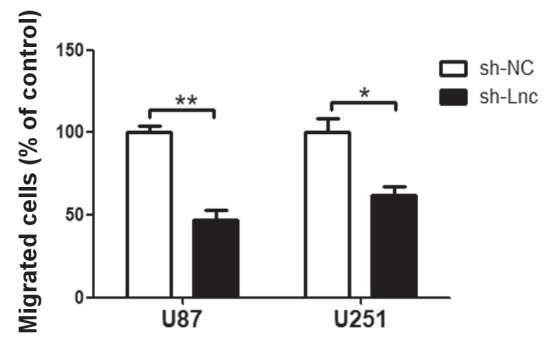

C
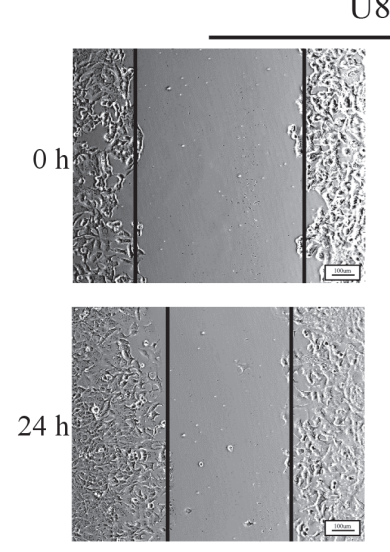

sh-NC
U87
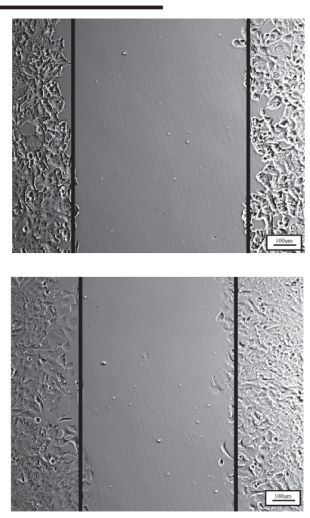

sh-Lnc

\section{B}

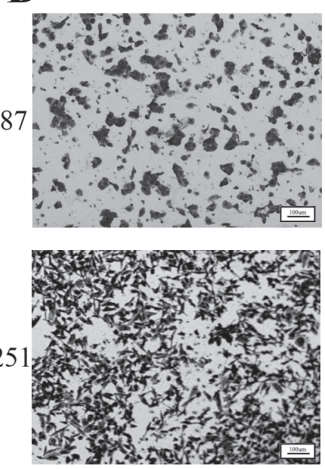

sh-NC
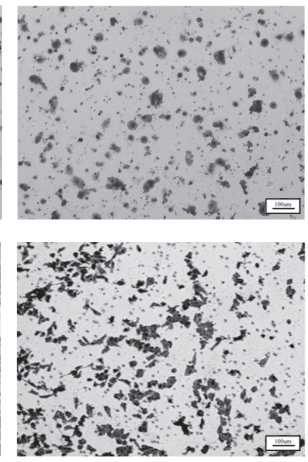

sh-Lnc

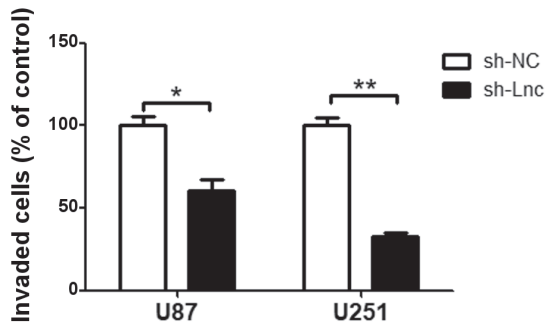

$\mathrm{U} 251$

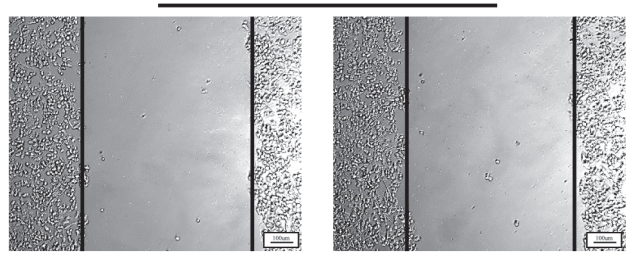

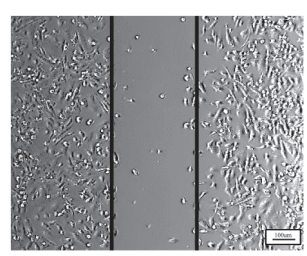

sh-NC

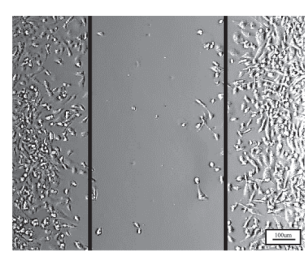

sh-Lnc
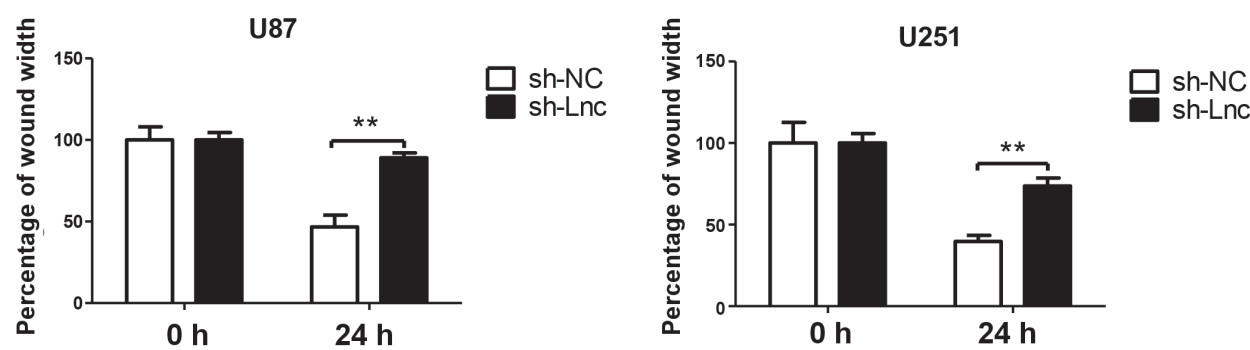

Figure 3. Knock-down of HOXB-AS1 suppressed migration and invasion of GBM cells. A) Cell migration was measured by Transwell migration assay in U87 and U251 cells transfected sh-NC and sh-Lnc. HOXB-AS1 significantly suppressed migration of GBM cells. B) Cell invasion was measured by Transwell invasion assay in U87 and U251 cells transfected sh-NC and sh-Lnc. HOXB-AS1 significantly suppressed invasion of GBM cells. C) Migration distance was evaluated by scratch wound assay, and HOXB-AS1 knock-down significantly decreased the migration distance in both cell lines. ${ }^{\star} \mathrm{p}<0.05,{ }^{* *} \mathrm{p}<0.01$. 
A
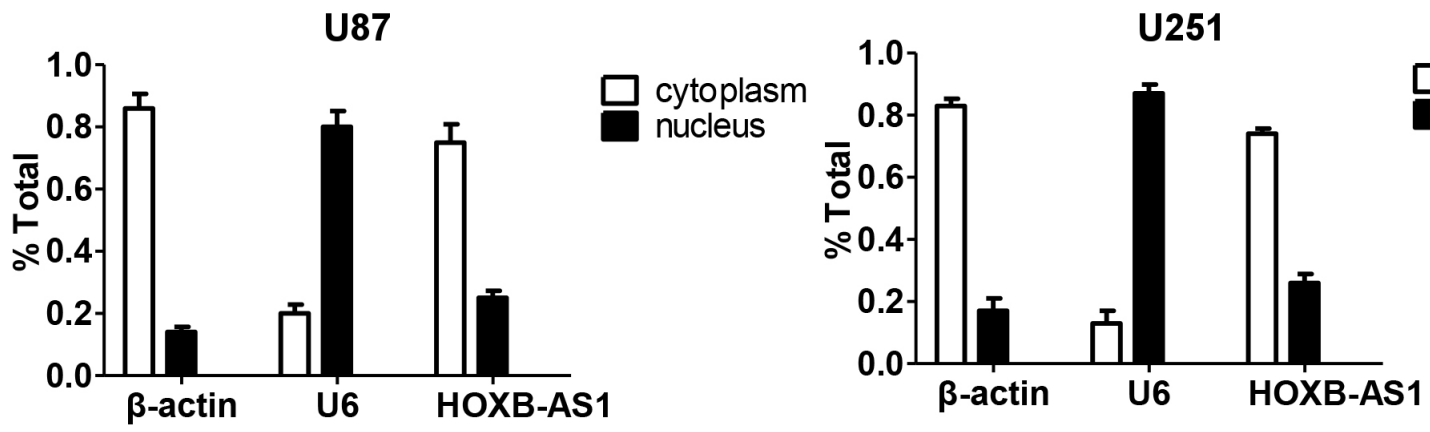

B

C

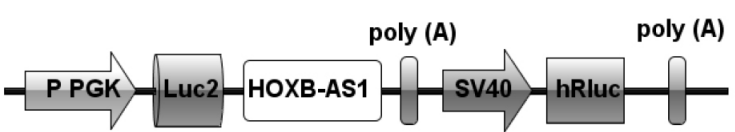

HOXB-AS1 5'...GCCACCGC--CCC $\overline{C G C T G C C A} \ldots 3^{\prime}$ |||||||||||||||||| hsa-miR-885-3p ATAGGTGATGTGGGGCGACGGA

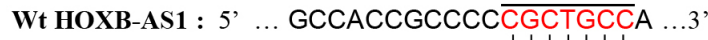
Mut HOXB-AS1: 3 '... GCCACCGCCCCATAGTAAA ... 5'

D

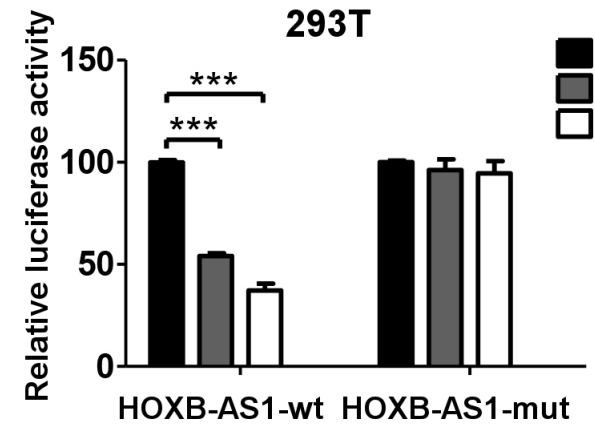

F

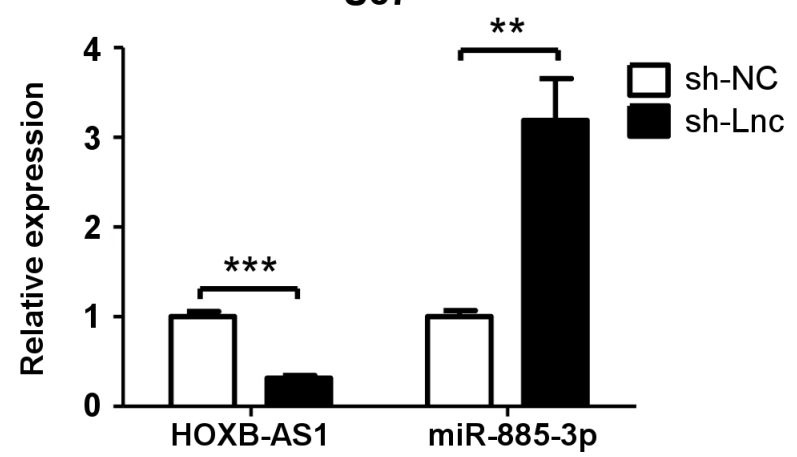

$\mathbf{E}$

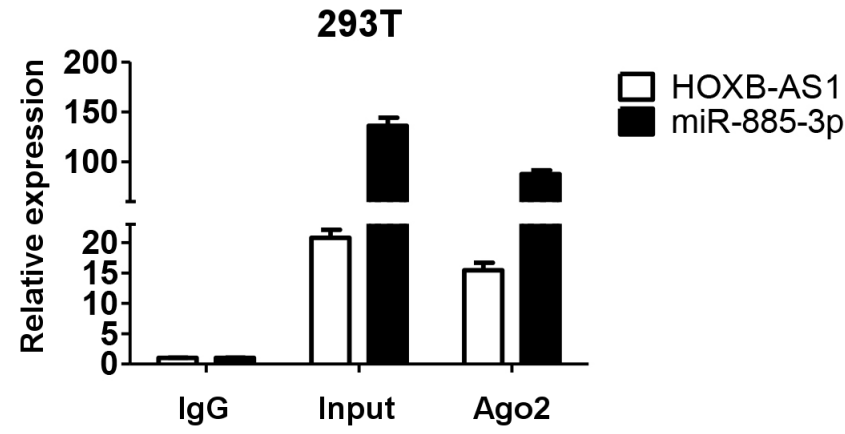

U251

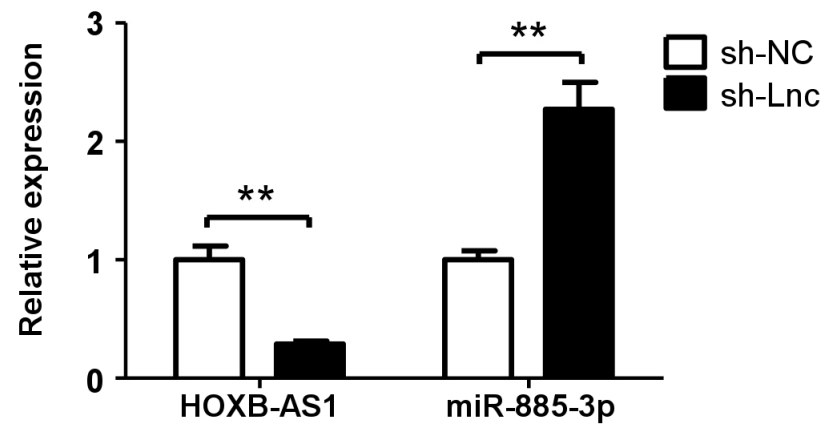

Figure 4. HOXB-AS1 functioned as ceRNA via directly sponging miR-885-3p. A) RNA in nucleus and plasma was separately extracted, and the subcellular location of HOXB-AS1 was examined. B) The predicted targeting sequence of miR-885-3p on the HOXB-AS1. C) The wild-type and mutant type of binding sites between HOXB-AS1 and miR-885-3p. D) HEK293T cells were transfected with HOXB-AS1 Wt or Mut and miR-885-3p. Luciferase reporter assay revealed the combination between miR-885-3p and HOXB-AS1. E) Anti-Ago2 RIP assay confirmed the combination between miR-8853p and HOXB-AS1. F) The expression of miR-885-3p after knock-down of HOXB-AS1 in both cell lines. ${ }^{* *} \mathrm{p}<0.01,{ }^{* * *} \mathrm{p}<0.001$. 
A

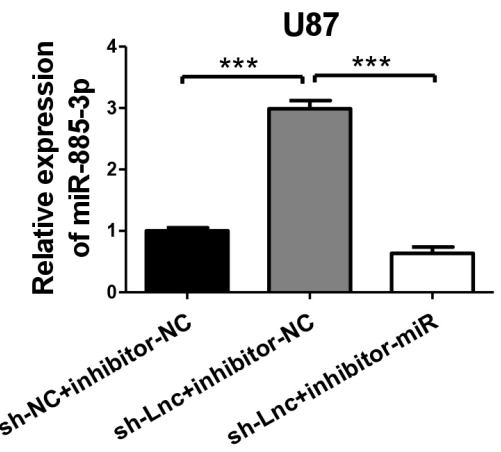

B

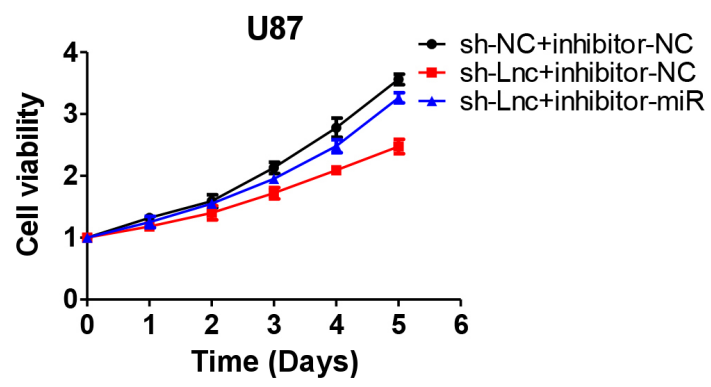

C

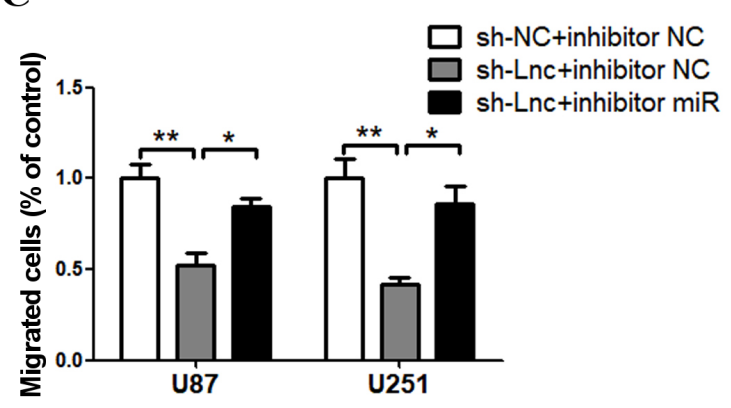

D

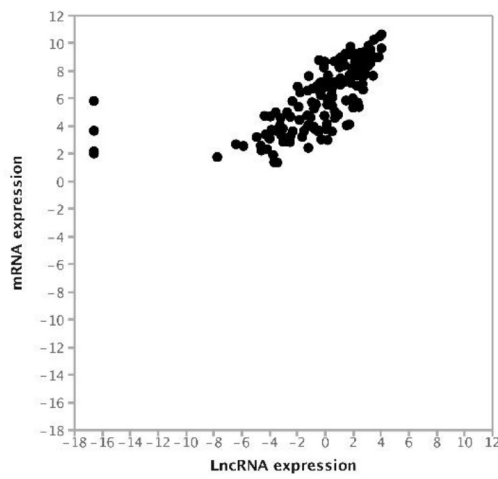

hsa-miR-885-3p ATAGGTGATGTGGG $\overline{\text { GCGACGGA }}$

HOXB2 3' UTR 5'...TGAGAAATGUGGCGCTGCCG...3'

$\mathbf{E}$

F

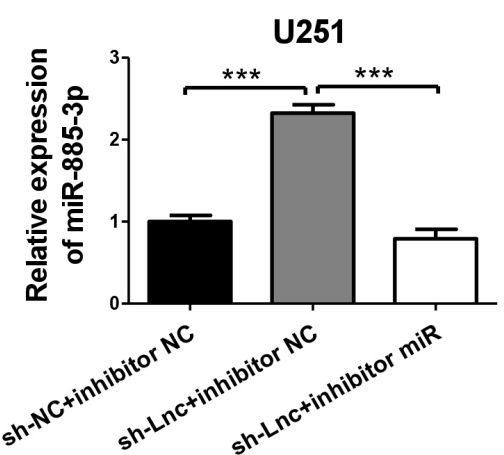

U251
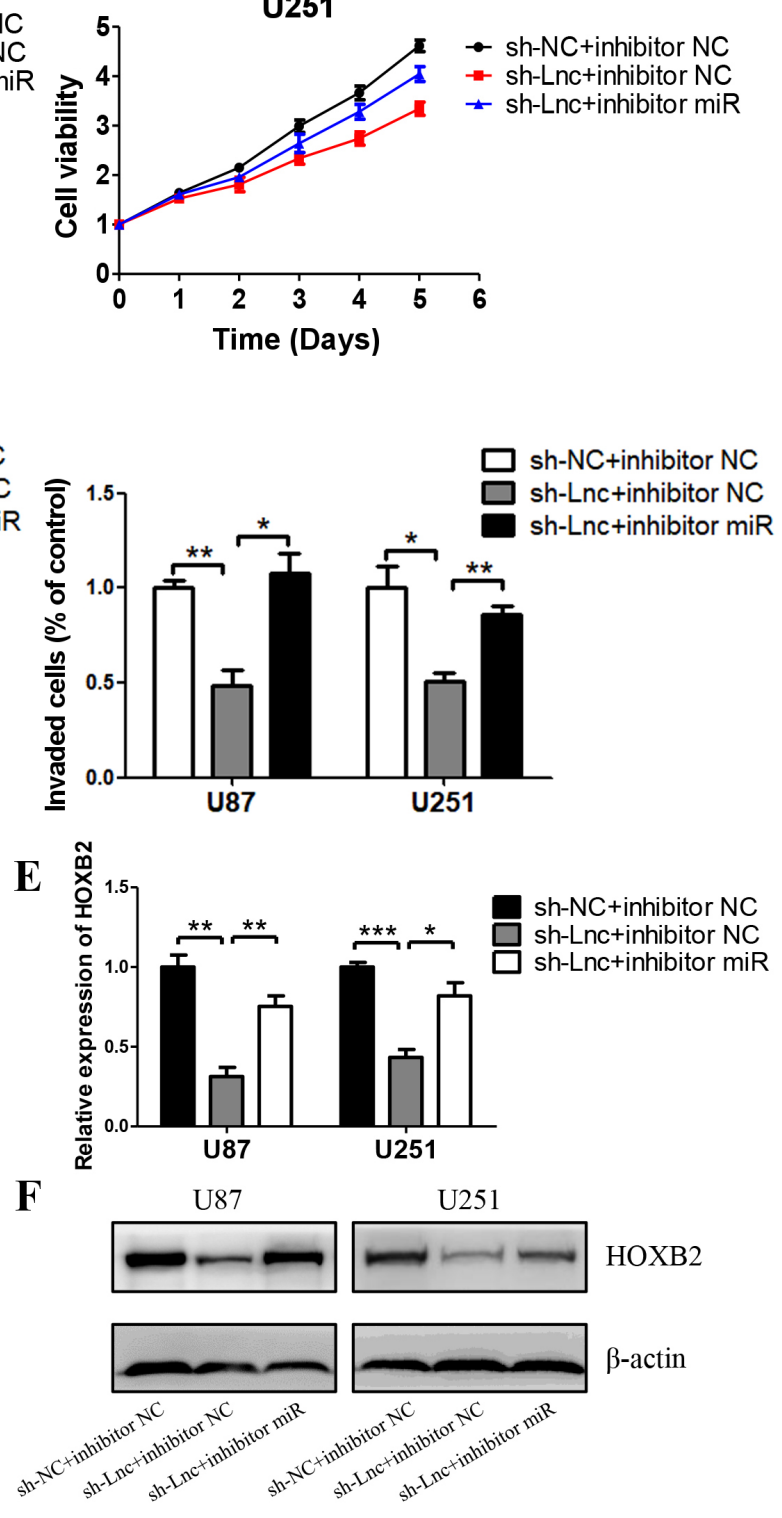

Figure 5. HOXB-AS1 promoted the proliferation and migration of GBM cells via HOXB-AS1/miR-885-3p/HOXB2 axis. A) Expression of miR-885-3p after HOXB-AS1 knock-down and miR-885-3p inhibition. B) MiR-885-3p rescued the proliferation inhibition effect of HOXB-AS1 knock-down. C) MiR-885-3p rescued the migration and invasion inhibition effects of HOXB-AS1 knock-down. D) Correlation between HOXB-AS1 and HOXB2 expression based on TCGA database. E, F) Expression of HOXB2 after HOXB-AS1 knock-down and miR-885-3p inhibition on mRNA and protein levels. ${ }^{*} \mathrm{p}<0.05,{ }^{* *} \mathrm{p}<0.01,{ }^{* * *} \mathrm{p}<0.001$. 
To find the target genes of HOXB-AS1, we firstly analyzed the correlation between the expression of HOXB-AS1 and genes in GBM tissues based on TCGA database. As shown in Figure 5D, we found that HOXB2 was positively correlated with HOXB-AS1, suggesting that HOXB2 might be a potential target of HOXB-AS1. Also, we found that HOXB2 was a potential target of miR-885-3p, indicating that HOXB-AS1 might function via HOXB2/ miR-885-3p pathway. qRT-PCR and western blot showed that knock-down of HOXB-AS1 suppressed HOXB2 and inhibition of miR-885-3p could rescue the expression level in both GBM cell lines, which further demonstrated that HOXB-AS1 could function via HOXB-AS1/miR-885-3p/ HOXB2 axis.

\section{Discussion}

In glioblastoma, a cluster of lncRNAs has been reported to be essential for tumor development. For example, lncRNA H19 can positively affect the tumorigenic properties of glioblastoma cells [27]. Knock-down of SOX2OT inhibited malignant biological behavior of glioblastoma stem cells [28]. It is of great significance to further explore the functions and mechanisms of lncRNAs. In our study, we downloaded and analyzed the RNA-seq data and clinical information from TCGA database and we showed that an uncharacteristic IncRNA HOXB-AS1 was significantly up-regulated in GBM tissues compared to LGG tissues (Figure 1). HOXB-AS1 was also overexpressed in glioblastoma cells compared to normal HA cells, which further suggested the important functions in tumorigenesis and progression of GBM (Figure 1C). Moreover, we found that patients with high expression level of HOXB-AS1 had poorer prognosis in both LGG and GBM groups (Figure 1D). In conclusion, our findings indicate that HOXB-AS1 is a functional and prognostic lncRNAs which might regulate malignant behavior of GBM cells.

As high speed of proliferation and metastasis of GBM cells was a key factor influencing the prognosis of patients [29], we firstly evaluated the effect of HOXB-AS1 on cell proliferation, migration and invasion. As shown in Figure 2, we found that knock-down of HOXB-AS1 significantly suppressed GBM cell proliferation. Further, flow cytometry data showed that inhibition of HOXB-AS1 induced remarkable G2 phase arrest in both U87 and U251 cell lines, which explained the mechanism of proliferation suppression. To evaluate the influence of HOXB-AS1 on metastatic ability of GBM cells, transwell assay and wound healing assay were performed. We found that the migrated and invaded cells and wound width reparation were suppressed in HOXB-AS1 knock-down group, which proved that HOXB-AS1 could also promote migration of the GBM cells (Figure 3). Our findings demonstrate that HOXB-AS1 is a functional lncRNA which can regulate both proliferation and metastasis of GBM cells.
After showing the functions of HOXB-AS1 in GBM cells, we further explored the mechanisms. Previous studies reported that IncRNAs could function via chromatin remodeling, alternative splicing, mRNA stability, and miRNA sponge, which might closely correlate with subcellular locations of lncRNAs [13]. As shown in Figure 4, we found that HOXB-AS1 was mainly located in the cytoplasm of cells, which indicates that it might act as ceRNA via sponging and blocking functions of miRNAs. Then, the miR-885-3p binding site was found in the HOXBAS1sequence based on bioinformatic analysis (Figure 4B). To verify the binding of miR-885-3p to HOXB-AS1, full length Wt or Mut HOXB-AS1 sequence was cloned into pMIR-GLO vector. The luciferase reporter assay showed that miR-885-3p could directly combine with HOXB-AS1 and anti-Ago2 RIP assay further confirmed this finding. Ago2 is a key component of RNA induced silencing complexes (RISC) [30]. We detected expression correlation between HOXB-AS1 and miR-885-3p. We found that knock-down of HOXB-AS1 increased miR-885-3p expression level in both U87 and U251 cell lines, which further proved the existence of HOXB-AS1/ miR-885-3p/Ago2 complex.

After showing that HOXB-AS1 could function via sponging miR-885-3p, we further verified whether miR-885-3p inhibition could rescue the proliferation, migration and invasion suppression caused by HOXB-AS1 knock-down. As shown in Figure 5, we found that knockdown of HOXB-AS1 significantly suppressed proliferation, migration and invasion while addition of miR-885-3p inhibitor rescued it. As miRNAs may execute their functions via degradation and/or blocking of translation of the mRNAs, we found that HOXB2, a gene that was predicted to be positively correlated with HOXB-AS1 expression, could be targeted by miR-885-3p. Further, qRT-PCR and western blot also confirmed HOXB2 as a target of miR-885-3p (Figures 5E, 5F). Our results demonstrate that miR-885-3p is a functional target of HOXB-AS1 via inhibiting HOXB2 expression.

In conclusion, we discovered a novel lncRNA termed HOXB-AS1 that regulates proliferation, migration and invasion of GBM cells via HOXB-AS1/miR-885-3p/HOXB2 axis. Our research broadens our insights into the underlying mechanisms in carcinogenesis and progression of GBM and provides a potential biomarker for clinical diagnosis and treatment of GBM patients.

Supplementary information is available in the online version of the paper.

Acknowledgements: This work was supported by grants from the Natural Science Foundation of Jiangsu Province (No. BK20161174), Jiangsu Key Laboratory of New Drug Research and Clinical Pharmacy (KF-XY201503), Six Talent Peaks Project in Jiangsu Province (No. 2015-SWYY-018), and Innovation Project for Graduate Student of Jiangsu Province (No.SJZZ16-0289). 


\section{References}

[1] CANCER GENOME ATLAS RESEARCH NETWORK. Comprehensive genomic characterization defines human glioblastoma genes and core pathways. Nature 2008; 455: 1061-1068. https://doi.org/10.1038/nature07385

[2] OSTROM QT, GITTLEMAN H, XU J, KROMER C, WOLINSKY Y et al. CBTRUS Statistical Report: Primary Brain and Other Central Nervous System Tumors Diagnosed in the United States in 2009-2013. Neuro-oncology 2016; 18: v1-v75. https://doi.org/10.1093/neuonc/now207

[3] POLIVKA J JR, POLIVKA J, ROHAN V, TOPOLCAN O, FERDA J. New molecularly targeted therapies for glioblastoma multiforme. Anticancer Res 2012; 32: 2935-2946.

[4] PREUSSER M, DE RIBAUPIERRE S, WOHRER A, ERRIDGE SC, HEGI M et al. Current concepts and management of glioblastoma. Ann Neurol 2011; 70: 9-21. https://doi. org/10.1002/ana.22425

[5] STUPP R, MASON WP, VAN DEN BENT MJ, WELLER M, FISHER B et al. Radiotherapy plus concomitant and adjuvant temozolomide for glioblastoma. N Engl J Med 2005; 352: 987-996. https://doi.org/10.1056/NEJMoa043330

[6] BAI RY, STAEDTKE V, RIGGINS GJ. Molecular targeting of glioblastoma: Drug discovery and therapies. Trends Mol Med 2011; 17: 301-312. https://doi.org/10.1016/j.molmed.2011.01.011

[7] HADZIAHMETOVIC M, SHIRAI K, CHAKRAVARTI A. Recent advancements in multimodality treatment of gliomas. Future Oncol 2011; 7: 1169-1183. https://doi.org/10.2217/ fon. 11.102

[8] Brodie S, Lee HK, Jiang W, Cazacu S, Xiang C et al. The novel long non-coding RNA TALNEC2, regulates tumor cell growth and the stemness and radiation response of glioma stem cells. Oncotarget 2017; 8: 31785-31801. https://doi. org/10.18632/oncotarget.15991

[9] LIU S, MITRA R, ZHAO MM, FAN W, EISCHEN CM et al. The Potential Roles of Long Noncoding RNAs (lncRNA) in Glioblastoma Development. Mol Cancer Ther 2016; 15: 2977-2986. https://doi.org/10.1158/1535-7163.MCT-160320

[10] YUE M, CHARLES RICHARD JL, OGAWA Y. Dynamic interplay and function of multiple noncoding genes governing $\mathrm{X}$ chromosome inactivation. Biochim Biophys Acta 2016; 1859: 112-120. https://doi.org/10.1016/j. bbagrm.2015.07.015

[11] BUTLER AA, WEBB WM, LUBIN FD. Regulatory RNAs and control of epigenetic mechanisms: expectations for cognition and cognitive dysfunction. Epigenomics 2016; 8: 135-151. https://doi.org/10.2217/epi.15.79

[12] CHAILLET JR. Genomic imprinting: lessons from mouse transgenes. Mutat Res 1994; 307: 441-449. https://doi. org/10.1016/0027-5107(94)90255-0

[13] QUAN M, CHEN J, ZHANG D. Exploring the secrets of long noncoding RNAs. Int J Mol Sci 2015; 16: 5467-5496. https://doi.org/10.3390/ijms16035467

[14] LI Y, LIANG Y, SANG Y, SONG X, ZHANG H et al. MiR770 suppresses the chemo-resistance and metastasis of triple negative breast cancer via direct targeting of STMN1. Cell Death Dis 2018; 9: 14. https://doi.org/10.1038/s41419-0170030-7
[15] YANG C, ZHENG J, XUE Y, YU H, LIU X et al. The Effect of MCM3AP-AS1/miR-211/KLF5/AGGF1 Axis Regulating Glioblastoma Angiogenesis. Front Mol Neurosci 2017; 10: 437. https://doi.org/10.3389/fnmol.2017.00437

[16] SHI J, WANG YJ, SUN CR, QIN B, ZHANG Y et al. Long noncoding RNA lncHERG promotes cell proliferation, migration and invasion in glioblastoma. Oncotarget 2017; 8: 108031-108041. https://doi.org/10.18632/oncotarget.22446

[17] LI J, HAN L, ROEBUCK P, DIAO L, LIU L et al. TANRIC: An Interactive Open Platform to Explore the Function of lncRNAs in Cancer. Cancer Res 2015; 75: 3728-3737. https:// doi.org/10.1158/0008-5472.CAN-15-0273

[18] MA Z, PENG P, ZHOU J, HUI B, JI H et al. Long Non-Coding RNA SH3PXD2A-AS1 Promotes Cell Progression Partly Through Epigenetic Silencing P57 and KLF2 in Colorectal Cancer. Cell Physiol Biochem 2018; 46: 2197-2214. https:// doi.org/10.1159/000489589

[19] LIANG Y, SONG X, LI Y, SANG Y, ZHANG N et al. A novel long non-coding RNA-PRLB acts as a tumor promoter through regulating miR-4766-5p/SIRT1 axis in breast cancer. Cell Death Dis 2018; 9: 563. https://doi.org/10.1038/ s41419-018-0582-1

[20] LIVAK KJ, SCHMITTGEN TD. Analysis of relative gene expression data using real-time quantitative PCR and the 2(-Delta Delta C(T)) method. Methods 2001; 25: 402-408. https://doi.org/10.1006/meth.2001.1262

[21] KE SB, QIU H, CHEN JM, SHI W, CHEN YS. MicroRNA202-5p functions as a tumor suppressor in colorectal carcinoma by directly targeting SMARCC1. Gene 2018; 676: 329-335. https://doi.org/10.1016/j.gene.2018.08.064

[22] YANG X, WANG M, LIN B, YAO D, LI J et al. miR-487a promotes progression of gastric cancer by targeting TIA1. Biochimie 2018; 154: 119-126. https://doi.org/10.1016/j.biochi.2018.08.006

[23] NAKADA M, ANDERSON EM, DEMUTH T, NAKADA S, REAVIE LB et al. The phosphorylation of ephrin-B2 ligand promotes glioma cell migration and invasion. Int J Cancer 2010; 126: 1155-1165. https://doi.org/10.1002/ijc.24849

[24] ZHANG Y, LIN C, LIAO G, LIU S, DING J et al. MicroRNA-506 suppresses tumor proliferation and metastasis in colon cancer by directly targeting the oncogene EZH2. Oncotarget 2015; 6: 32586-32601. https://doi.org/10.18632/oncotarget.5309

[25] CHANG YY, YEN CJ, CHAN SH, CHOU YW, LEE YP et al. NEK2 Promotes Hepatoma Metastasis and Serves as Biomarker for High Recurrence Risk after Hepatic Resection. Ann Hepatol 2018; 17: 843-856. https://doi. org/10.5604/01.3001.0012.3146

[26] IKEDA K, SATOH M, PAULEY KM, FRITZLER MJ, REEVES WH et al. Detection of the argonaute protein Ago2 and microRNAs in the RNA induced silencing complex (RISC) using a monoclonal antibody. J Immunol Methods 2006; 317: 38-44. https://doi.org/10.1016/j.jim.2006.09.010

[27] FAZI B, GARBO S, TOSCHI N, MANGIOLA A, LOMBARI $\mathrm{M}$ et al. The lncRNA H19 positively affects the tumorigenic properties of glioblastoma cells and contributes to NKD1 repression through the recruitment of EZH2 on its promoter. Oncotarget 2018; 9: 15512-15525. https://doi.org/10.18632/ oncotarget. 24496 
[28] SU R, CAO S, MA J, LIU Y, LIU X et al. Knock-down of SOX2OT inhibits the malignant biological behaviors of glioblastoma stem cells via up-regulating the expression of miR194-5p and miR-122. Mol Cancer 2017; 16: 171. https://doi. org/10.1186/s12943-017-0737-1
[29] LI P, WU M. Epigenetic Mechanisms of Glioblastoma, pp 43-58. In. De Vleeschouwer S (Eds.). Glioblastoma. Codon Publications, Brisbane 2017, p 452. ISBN: 978-0-9944381-2-6

[30] FILIPOWICZ W, BHATTACHARYYA SN, SONENBERG $\mathrm{N}$. Mechanisms of post-transcriptional regulation by microRNAs: are the answers in sight? Nat Rev Genet 2008; 9: 102-114. https://doi.org/10.1038/nrg2290 


\section{Long non-coding RNA HOXB-AS1 promotes proliferation, migration and invasion of glioblastoma cells via HOXB-AS1/miR-885-3p/HOXB2 axis}

X. CHEN, L. Q. LI, X. QIU, H. WU

Supplemental material
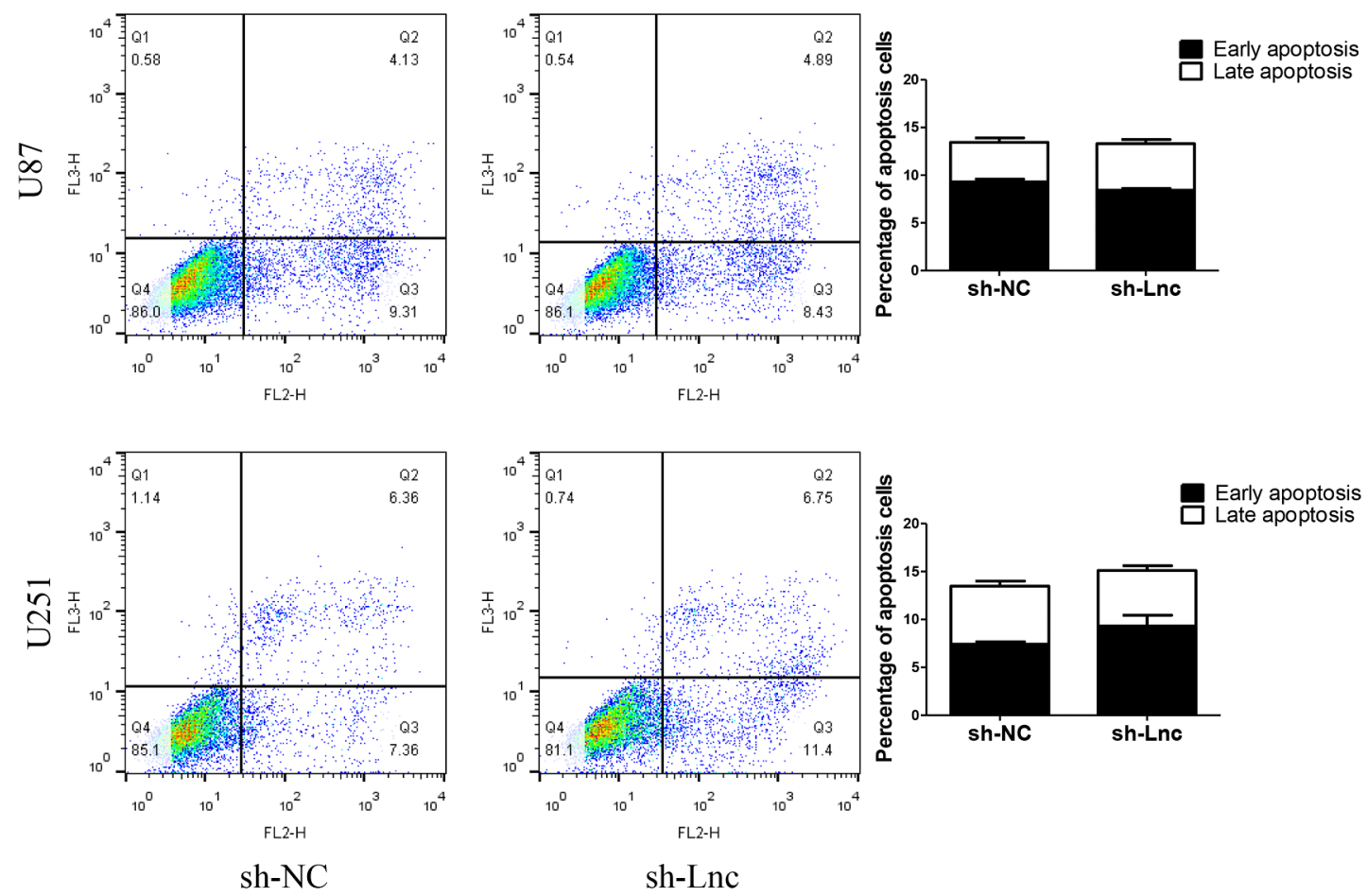

Supplementary Figure 1. Effect of HOXB-AS1 knock-down on apoptosis of GBM cell lines. 